archives-ouvertes

\title{
Adaptation of Saccadic Eye Movements: Transfer and Specificity
}

\author{
Nadia Alahyane, Denis Pelisson
}

\section{To cite this version:}

Nadia Alahyane, Denis Pelisson. Adaptation of Saccadic Eye Movements: Transfer and Specificity. Annals of the New York Academy of Sciences, Wiley, 2003, 1004 (1), pp.1-9. 10.1196/annals.1303.001 . hal-02199453

\section{HAL Id: hal-02199453 \\ https://hal.archives-ouvertes.fr/hal-02199453}

Submitted on 31 Jul 2019

HAL is a multi-disciplinary open access archive for the deposit and dissemination of scientific research documents, whether they are published or not. The documents may come from teaching and research institutions in France or abroad, or from public or private research centers.
L'archive ouverte pluridisciplinaire HAL, est destinée au dépôt et à la diffusion de documents scientifiques de niveau recherche, publiés ou non, émanant des établissements d'enseignement et de recherche français ou étrangers, des laboratoires publics ou privés. 


\title{
Adaptation of Saccadic Eye Movements: Transfer and Specificity
}

\author{
NADIA ALAHYANE AND DENIS PÉLISSON \\ “Espace et Action” INSERM U534, 16 avenue du doyen Lépine, \\ 69500 Bron, France
}

\begin{abstract}
The present study was designed to test whether the adaptation of saccadic eye movements depends only on the eye displacement vector of the trained saccade or also on eye position information. Using the double target step paradigm 1 in eight human subjects, we first induced in a single session two "opposite directions adaptations" (ODA) of horizontal saccades of the same vector. Each ODA (backward or forward) was linked to one vertical eye position $\left(12.5^{\circ}\right.$ up or $25^{\circ}$ down) and alternated from trial to trial. The results showed that opposite changes of saccade amplitude can develop simultaneously, indicating that saccadic adaptation depends on orbital eye position. This finding has important functional implications because in everyday life our eyes saccade from constantly changing orbital positions. A comparison of these data to two control conditions in which training trials of a single type (backward or forward) are presented at both $12.5^{\circ}$ and $-25^{\circ}$ eye elevations further indicated that eye position specificity is complete for backward, but not for forward, adaptation. Finally, the control conditions also indicated that the adaptation of a single saccade fully transferred to untrained saccades of the same vector, but initiated from different vertical eye positions. In conclusion, our study indicates that saccadic adaptation mechanisms use vectorial eye displacement signals, but can also take eye position signals into account as a contextual cue when the training involves conflicting saccade amplitude changes.
\end{abstract}

KEYWORDS: eye movement; human; adaptation; eye displacement vector; context

\section{INTRODUCTION}

Saccades are quick and accurate eye movements that constitute a good model for the study of sensorimotor plasticity. By using the double-step target paradigm pioneered by McLaughlin, ${ }^{1}$ both saccade amplitude increases or decreases can be elicited when the second, intrasaccadic, target step is directed in the direction of the first target step (forward adaptation) or opposite to it (backward adaptation), respectively. AU: Pls. give tel. \& fax nos. and e-mail for With this paradigm, a number of studies have analyzed the properties of saccadic adaptation. On the one hand, it was found that saccadic adaptation is specific to the amplitude and the direction of the trained saccade (vector-specific adaptation) because

Address for correspondence: Dr. Denis Pélisson,” Espace et Action” INSERM U534, 16 avenue du doyen Lépine, 69500 Bron, France.

pelisson@lyon.inserm.fr 
all saccades of the same vector as the adapted saccade-regardless of their starting orbital position-show a similar adaptive modification. ${ }^{2-5}$ On the other hand, Shelhamer and Clendaniel ${ }^{6}$ have suggested that eye position can serve as a contextual cue for saccadic adaptation. Indeed, they showed that two different adaptative states can develop concurrently in a single training session when forward and backward adaptation trials are associated with two different vertical eye positions. However, the conclusions of these authors are limited because (1) the number of tested subjects (four) is small given the known intersubject variability of saccadic adaptation, (2) the effectiveness of context-specific adaptations was evaluated indirectly and in only one of the four subjects, and (3) the two different types of adaptations were not presented randomly in interleaved trials but in alternating blocks of 20 identical trials. For these reasons, we designed a study measuring in the same group of 8 subjects both the simultaneous acquisition of two conflicting adaptations and the transfer of a single saccade adaptation to saccades initiated from untrained locations. In the main condition ("opposite directions adaptations," ODA), both backward and forward adaptations of horizontal saccades were elicited in a single training session, each direction of adaptation being related to a different eye elevation. This was compared with two control conditions ("same direction adaptations"), in which the same training (backward or forward) was performed with the eyes deviated up and down.

\section{MATERIALS AND METHODS}

\section{Experimental Device}

Subjects sat in a dimly illuminated room, with their head immobilized by a biteboard. They looked at red light-emitting diodes (LEDs) located on a concave spherical target board (distance $1.1 \mathrm{~m}$ ) along four horizontal meridians (meridians 1 to 4 : elevation of $12.5^{\circ}, 0^{\circ},-12.5^{\circ}$, and $-25^{\circ}$, respectively).

\section{Experimental Protocol}

The three experimental conditions each comprised a pretest session (40 trials), an adaptation session (240 trials), and a posttest session (40 trials). The (ODA) condition was always performed first. It was followed by the same direction backward adaptation (SDBA) and the same direction forward adaptation (SDFA) conditions carried out in a random order according to subjects. Each condition was done on a separate day.

Adaptation session. At the beginning of a trial, the subject looked at a fixation point (FP) located in the left hemifield at a distance of $12.5^{\circ}$ from the center. After a 1200 ms delay, FP was turned off while a target (T1) was simultaneously illuminated in the right hemifield at a $18.75^{\circ}$ location ( step $1=31.25^{\circ}$ ). During the saccade to step 1 (primary saccade), T1 was replaced by another target (T2) located $6.25^{\circ}$ away (step $2=20 \%$ of step 1). Step 2 was leftward (backward relative to step 1) in backward adaptation and rightward in forward adaptation. In the ODA condition, for four subjects (see FIG. 1A), target T2 stepped forward when the subject's eyes were directed at meridian 1 and stepped backward when the subject's eyes were directed at meridian 4, according to a random sequence; this pattern was reversed for the other 

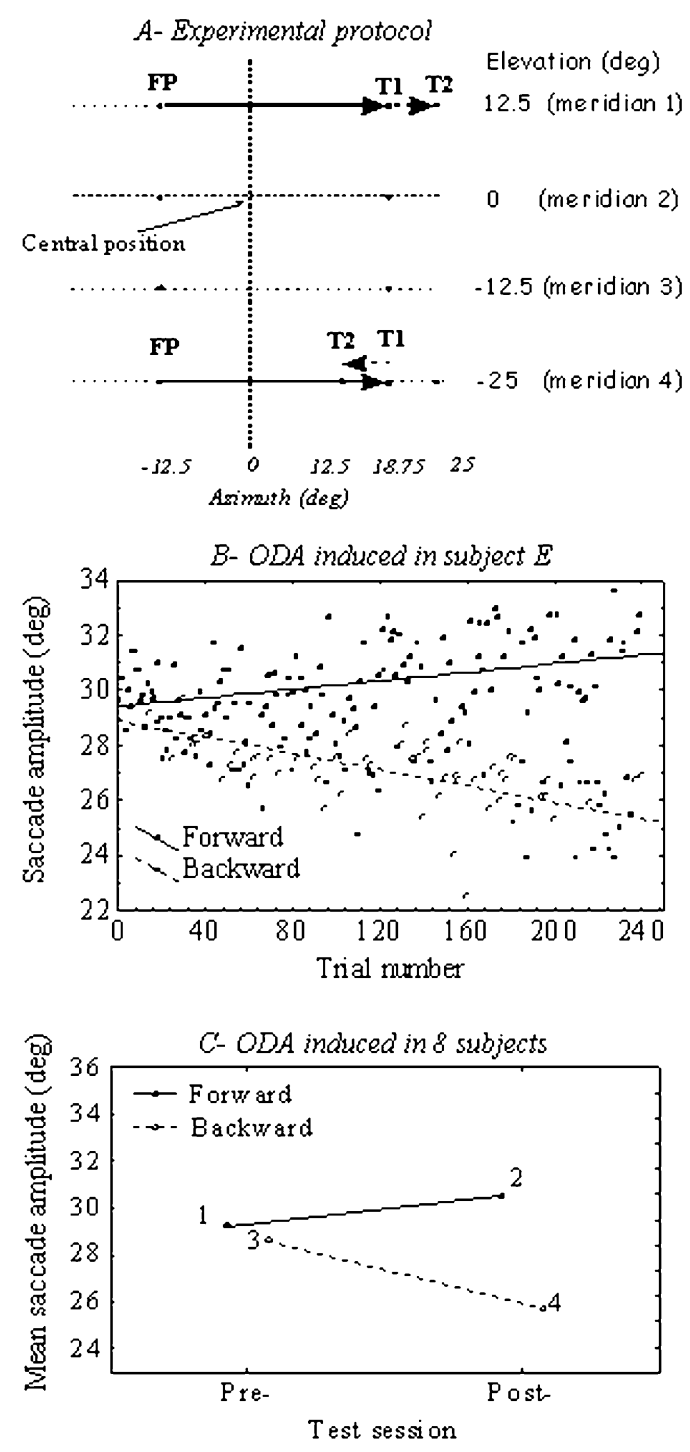

FIGURE 1. Opposite directions adaptation (ODA). (A) Experimental protocol showing the double target stimulations along meridians 1 and 4 used in four subjects to simultaneously produce forward and backward adaptations with the eyes deviated $12.5^{\circ}$ up or $25^{\circ}$ down, respectively. The reverse pattern was used in the other four subjects. FP: fixation point; T1: target 1; T2: target 2; step 1: $\mathrm{FP} \rightarrow \mathrm{T} 1\left(31.25^{\circ}\right)$; step 2: $\mathrm{T} \rightarrow \mathrm{T} 2\left(6.25^{\circ}\right)$. (B) Plots of saccade amplitude versus trial number during the adaptation session for backward (open circles) and forward saccadic adaptation (filled circles) induced in subject E. Linear regressions shown by a broken (backward; $\mathrm{R}=-0.68, P<0.001$ ) or a continuous line (forward; $\mathrm{R}=0.38, P$ $<0.001$ ). (C) Mean saccade amplitude recorded during pre- and posttest sessions for forward and backward adaptations (results of the two-way repeated measures ANOVA in text) (8 subjects). 
four subjects. In the two SDA conditions, step 2 was the same regardless of the meridian (1 or 4) and was directed to the left in the SDBA condition or to the right in the SDFA condition.

Pretest and posttest sessions. The target made a single step from FP $\left(-12.5^{\circ}\right)$ to $\mathrm{T} 1\left(18.75^{\circ}\right)$, which was extinguished at the beginning of the saccadic response. This single step occurred along meridians 1 and 4 as in the adaptation sessions, but also along meridians 2 and 3 to test the existence of transfer of adaptation to these two "untrained" meridians in SDBA and SDFA conditions.

\section{Eye Movement Recording and Data Analysis}

Horizontal and vertical eye movements were recorded by an eye-link system cities of (SMI, Germany). A PC program (DataWave, USA) controlled the experiment and recorded to disk eye-position signals. An electronic circuit fed by the left-eye position signal triggered the computer routine during the primary saccade to displace target T1 to T2 (step 2) during adaptation sessions and to switch it off during test sessions. The start and end of each saccade were detected offline on the basis of a velocity threshold of $40 \% \mathrm{~s}$ and corrected manually if necessary. The horizontal amplitude of each primary saccade of the left eye was calculated as the difference between initial and final eye positions. The time course of adaptation was evaluated by fitting a linear regression to the relationship between primary saccade amplitude and trial number. The percent saccade amplitude change between the posttest and the pretest sessions was calculated as follows: $100 *$ [(posttest amplitude - pretest amplitude) / pretest amplitude]. Finally, in both SDBA and SDFA conditions, the mean rate of adaptation transfer was computed separately for meridians 2 and 3 as follows: 100 * [mean amplitude change of untrained saccades (meridian 2 or 3)/mean amplitude change of trained saccades (meridians 1 and 4)].

\section{Statistical Analyses}

These analyses were performed on the mean saccade amplitude recorded during test sessions. First, in the ODA condition, a two-way repeated measures ANOVA was designed with adaptation direction (backward vs. forward) and type of test session (pre vs. post) as within-subject factors, and vertical eye position (meridian 1 vs. meridian 4) as between-subject factor. Second, the data of the ODA and of the SDA protocols were compared by performing, for each direction (backward and forward) separately, a two-way repeated measures ANOVA with type of adaptation (ODA vs. SDA) and type of test session (pre vs. post) as within-subject factors and vertical eye position as between-subject factor.

\section{RESULTS}

\section{Opposite Directions Adaptation (ODA) Condition}

Adaptation session. FIGURE 1B illustrates representative relationships between saccade amplitude and number of trials for subject $\mathrm{E}$ who underwent a forward adaptation when her eyes were directed upward (meridian 1) and a backward adaptation when her eyes were directed downward (meridian 4). The statistically 
significant linear regressions show that saccade amplitude decreased for the backward down-looking trials and simultaneously increased for the forward up-looking condition. These data of subject $E$ were representative of the group of subjects since the 16 regressions (forward or backward $\times 8$ subjects) all showed a slope consistent with the direction of the adaptation and only two failed to reach statistical significance $(P=0.05)$.

Statistical analysis of amplitude changes in test sessions. The results of the twoway ANOVA (adaptation direction $\times$ type of test session) on the mean primary saccade amplitude indicated a significant difference between backward adaptation and forward adaptation $(P<0.01)$, but no significant difference between the two types of test session $(P=0.2)$. The "vertical eye position" independent factor had no significant effect $(P=0.6)$. There was a significant $(P<0.001)$ interaction between the adaptation direction and the type of test session, as illustrated in Figure 1C. Post-hoc Fisher LSD tests showed that saccades made after backward adaptation (FIG. 1C, 4) were significantly smaller in amplitude than saccades made in the other three cases $(P<0.05)$. Similarly, saccades performed after forward adaptation (FIG. 1C, 2) were significantly larger in amplitude than those performed in the other three cases $(P$ $<0.001)$. In conclusion, ODA induced simultaneously led to significant modifications of saccade amplitude in the direction specified by step 2, and irrespective of vertical eye position. Note that backward adaptation $(-9.6 \pm 6.9 \%)$ was stronger than forward adaptation $(4.6 \pm 4.6 \%)$.

\section{Opposite Directions Adaptation vs. Same Direction Adaptation Conditions}

We then compared the ODA and control (SDA) conditions for backward and forward adaptations separately. For backward adaptation (FIG. 2A), the two conditions induced a similar change of saccade amplitude of about $12 \%$. The two-way ANOVA (type of adaptation $\times$ type of test session) revealed a significant difference between the two types of test sessions $(P<0.01)$, but not between the two types of backward adaptations $(P=0.2)$. Moreover, no significant interaction between the two factors $(P=0.8)$ was found. For forward adaptation (FIG. 2B), the adaptation was more effective when induced in the SDA condition than in the ODA situation (mean amplitude changes: $13 \pm 5.6 \%$ vs. $4.6 \pm 4.6 \%$, respectively). The ANOVA indeed revealed a significant difference between the two types of test session $(P<0.01)$, no significant difference between the two types of forward adaptations $(P=0.09)$, but a significant interaction between the two factors $(P<0.01)$. Post-hoc Fisher LSD tests indicated that the mean saccade amplitude in the posttest SDA condition (FIG. 2B, $4)$ was significantly larger than in the other three cases $(P<0.01)$. Similarly, the saccade amplitude in the posttest ODA condition (FIG. 2B, 2) was significantly different from the other three values $(P<0.05)$. Last, for both backward and forward adaptations, the vertical eye position independent factor had no significant effect $(P=0.3$ and $P=0.5$, respectively). This suggests that the pattern of results described above was independent of the meridian along which subjects actually experienced backward or forward adaptation in the ODA condition. In conclusion, the analyses of the present section indicate that when performed simultaneously in a single session, backward and forward adaptations interacted asymmetrically at the expense of forward adaptation. 


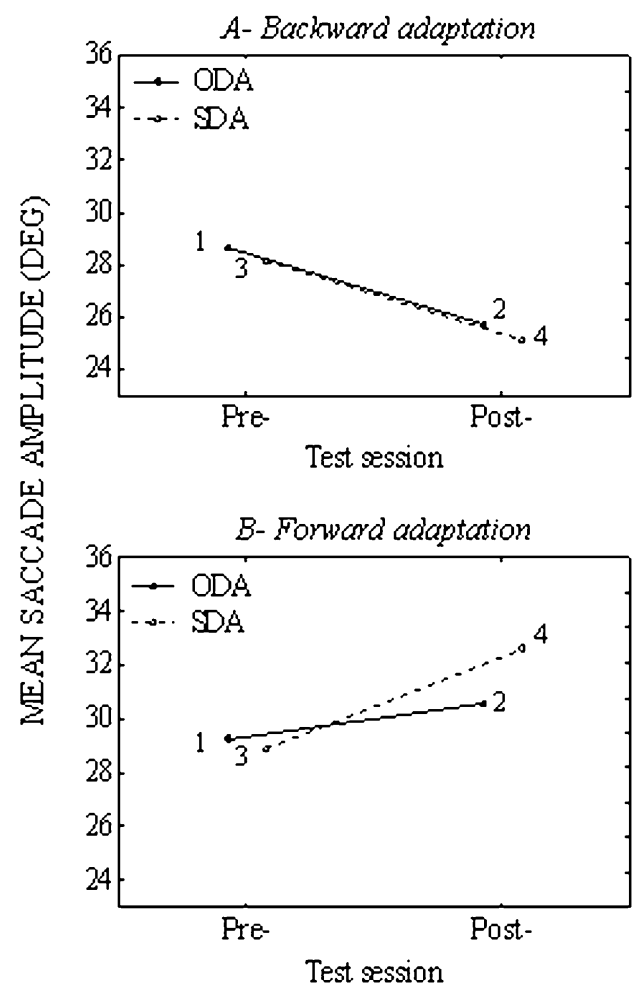

FIGURE 2. Comparison of opposite directions adaptation (ODA) to same direction adaptation (SDA) conditions (8 subjects). Plots of mean saccade amplitude recorded during the test sessions (pre vs. post) for ODA and SDA (results of the two-way repeated measures ANOVAs in text). (A) Backward adaptation. (B) Forward adaptation.

\section{Adaptation Transfer in Same Direction Adaptation Conditions}

In SDA conditions, the adaptation achieved along meridians 1 and 4 transferred to untrained saccades made along the two intermediate meridians (see FIG. 3). In addition, both for backward and forward adaptations, the transfer to these two untrained meridians was similar. Indeed, for backward adaptation, the transfer rate to meridian $2(102 \pm 21 \%)$ was not statistically different from the transfer rate to meridian $3(91 \pm 27 \%$; paired $t$ test, $P=0.2)$. Similarly, for forward adaptation, the transfer rates were again statistically indistinguishable (104 $\pm 25 \%$ vs. $100 \pm 32 \%$; paired $t$ test, $P=0.8$ ). These results indicate a complete adaptation transfer from saccades made along trained meridians ( 1 and 4 ) to untrained saccades (meridians 2 and 3$)$. 


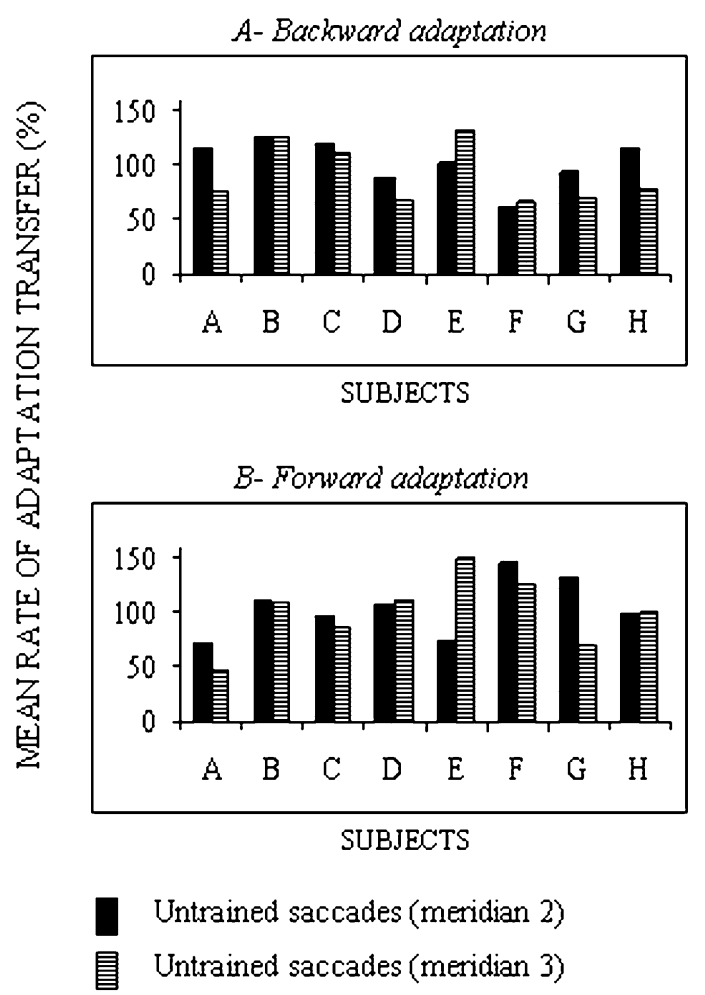

FIGURE 3. Transfer of adaptation from trained saccades to untrained saccades in the same direction adaptation (SDA) conditions (8 subjects). The rate of adaptation transfer from trained saccades performed along meridians 1 and 4 to untrained saccades elicited on intermediate meridians 2 (black bars) or 3 (striped bars) is plotted in all subjects (A-H). (A) Backward adaptation. (B) Forward adaptation.

\section{DISCUSSION}

Our study shows that saccadic adaptation is eye position-specific. Indeed, subjects were able to switch from an amplitude increase adaptation to an amplitude decrease adaptation when varying vertical eye position. Importantly, this switch operates on the short term, i.e., every time vertical eye position changed between successive trials. On the other hand, and in agreement with previous studies, ${ }^{2-5}$ saccadic adaptation is vector-specific. Indeed, we observed, in SDA conditions, a complete transfer of adaptation to untrained saccades of the same vector (same amplitude and direction) as the two trained saccades, but initiated from intermediate vertical eye positions. In fact, these two experimental situations test different phenomena. The second situation, demonstrating the vector specificity of saccadic adaptation, is unnatural as far as a single adaptation occurs. Instead, the first situation provides a more stringent test of eye-position dependency based on conflicting adaptations as 
encountered in everyday life. We thus conclude that saccadic adaptation (1) is specific to the trained eye-saccade vector and (2) transfers to other eye positions when there is no competing training. Eye position is, however, taken into account when competing trainings are performed at different orbital eye positions. Therefore, both eye position and eye displacement signals are used by the saccade-adaptive mechanisms under natural conditions.

Our results also indicate that the eye-position dependency of forward saccadic adaptation is not complete. Indeed, forward adaptation was weakened when paired with backward adaptation as compared to when it was induced alone in the control session (saccade amplitude changes: $23 \%$ vs. $65 \%$, respectively). This illustrates the effect of a conflict with backward adaptation. This conflict is partial and asymmetrical because the backward adaptation was as efficient as when induced alone in the control session (saccade amplitude changes: $48 \%$ vs. $52 \%$, respectively). This conflict in favor of the backward adaptation is consistent with the differences found in the speed of adaptation (data not shown), and suggests that forward adaptation shows less eye-position specificity. This agrees with the hypothesis of a differential control of these two opposite types of adaptation (e.g., Ref. 7).

Shelhamer and Clendaniel ${ }^{6}$ have recently investigated the effect of vertical eye position on horizontal saccadic adaptation, and their conclusions are somewhat consistent with ours. However, contrary to us, they observed a slight saccade amplitude decrease with forward adaptation. Because it was not compared to a SDA condition, this result casts some doubt as to the eye-position specificity of forward adaptation. This difference between the two studies can be explained by the fact that in the Shelhamer and Clendaniel study the two opposite types of adaptation were performed at vertical eye positions closer to each other than in our study $\left(20^{\circ}\right.$ vs. $37.5^{\circ}$ vertical separation), leading to a stronger conflict. Furthermore, 760 training trials were used as compared to only 240 in the present study, revealing a possible trade-off for saccadic adaptation between training duration and eye-position selectivity. Another difference between our study and the previous one ${ }^{6}$ is that in our study forward and backward adaptation double-step stimuli alternated randomly across successive trials, whereas in the previous study ${ }^{6}$ stimuli were presented in blocks of 20 identical trials. Thus, our study directly demonstrates that eye position interacts with saccadic adaptation over the short term, a situation closer to that experienced in everyday life. Since vertical eye position alternated randomly during the training as well as the test phases, our results also indicate that eye-position information is necessary both during the acquisition of adaptation and the retention of the modified behavior.

The behavioral properties discussed in this paper should be taken into account to try to understand the neurophysiological processes underlying saccadic adaptation. It is now quite clear that the medial part of the cerebellum is involved in saccadic adaptation (e.g., Refs. 8-10). In addition, the cerebellum receives afferent input from extraocular muscles and oculomotor commands from the brainstem (see Ref. ${ }^{11}$ for references), which possibly provide both eye position and eye displacement signals. The cerebellum is therefore well situated to change adaptively saccade amplitude in an eye position-dependent manner. Future studies will have to determine which dynamic selection processes are used by the cerebellum to switch between different adapted states according to different contexts, such as eye position. 


\section{ACKNOWLEDGMENTS}

We thank the subjects for their participation in this study. We also thank Marcia Riley and Christian Urquizar for designing the data replay/parameter extraction software. Research was supported by INSERM U534.

\section{REFERENCES}

1. McLaughlin, S.C. 1967. Parametric adjustment in saccadic eye movements. Percept. Psychophys. 2: 359-362.

2. MilleR, J.M., T. ANSTIS, et al. 1981. Saccadic plasticity: parametric adaptive control by retinal feedback. J. Exp. Psychol. Hum. Percept. Perform. 7: 356-366.

3. Deubel, H. 1987. Adaptivity of gain and direction in oblique saccades. In Eye Movements: From Physiology to Cognition. J.K. O’Regan \& A. Levy-Schoen, Eds.: 181190. Elsevier/North-Holland. New York.

4. Frens, M.A. \& A.J. Van Opstal. 1994. Transfer of short term adaptation in human saccadic eye movements. Exp. Brain Res. 100: 293-306.

5. Albano, J.E. 1996. Adaptive changes in saccade amplitude: oculocentric or orbitocentric mapping? Vision Res. 36: 2087-2098.

6. Shelhamer, M. \& R.A. Clendaniel. 2002. Context-specific adaptation of saccade gain. Exp. Brain Res. 146: 441-450.

7. Deubel, H., W. Wolf, et al. 1986. Adaptive gain control of saccadic eye movements. Hum. Neurobiol. 5: 245-253.

8. Desmurget, M., D. PÉlisson, et al. 1998. Functional anatomy of saccadic adaptation in humans. Nat. Neurosci. 1: 524-528.

9. TAKAGI, M., D.S. ZeE, et al. 1998. Effects of lesions of the oculomotor vermis on eye movements in primate: saccades. J. Neurophysiol. 80: 1911-1931.

10. Barash, S., A. Melikyan, et al. 1999. Saccadic dysmetria and adaptation after lesions of the cerebellar cortex. J. Neurosci. 19: 10931-10939.

11. PÉlisson, D., L. Goffart, et al. 2003. Control of saccadic eye movements and combined eye/head gaze shifts by the medio-posterior cerebellum. In Progress in Brain Research: Neural Control of Space Coding and Action Production. C. Prablanc, D. Pélisson, et al., Eds.: 69-89. Elsevier. Amsterdam. 